\title{
Maternal Sepsis
}

World Health Organization

\section{Source}

World Health Organization. (2017). Statement on Maternal Sepsis.

Maternal sepsis is a life-threatening condition defined as organ dysfunction resulting from infection during pregnancy, childbirth, post-abortion, or postpartum period. 